\title{
Videographic GIS Database Documentation Project for Ft. Knox, Kentucky: Planning and Process
}

by R.G. Sullivan and M.C. Vogt

Reclamation Engineering and Geosciences Section, Energy Systems Division,

Argonne National Laboratory, 9700 South Cass Avenue, Argonne, Illinois 60439

June 1992

Work sponsored by United States Department of Defense, United States Army Corps of Engineers, Construction Engineering Research Laboratories 


\section{Contents}

Abstract.............................................................................. 1

1 Introduction.................................................................... 1

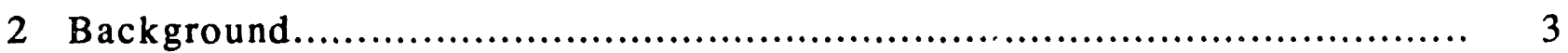

2.1 Computer Videographic Technology ....................................... 3

2.2 Videographic Applications in Army Training-Land Management ................ 4

3 Goal, Objectives, and Approach............................................ 5

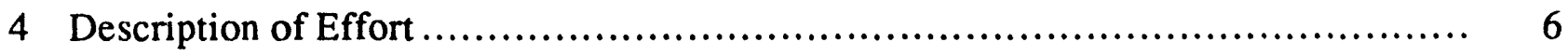

4.1 Development of the Project Work Plan ...................................... 6

4.2 Creation of GRASS Base Images......................................... 6

4.3 Conversion of GRASS Images to a Videographic-Compatible Format .......... 6

4.3.1 R.out.tga File Conversion............................................... 7

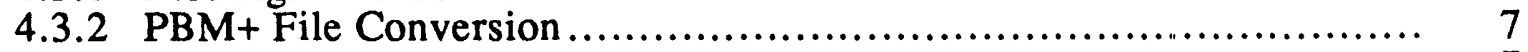

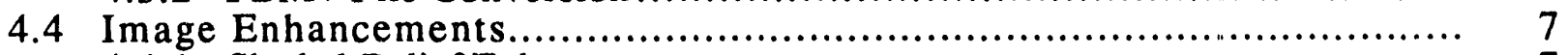

4.4.1 Shaded-Relief Enhancements ............................................ 7

4.4.2 Text and Labeling...................................................... 10

4.5 Creation of DOS- and Macintosh-Compatible Slide Shows ................... 11

4.5.1 Reduction of Image Color Depth.................................... 11

4.5.2 GIF File Format ...................................................... 11

4.5.3 Translation of Files to GIF Format.................................... 12

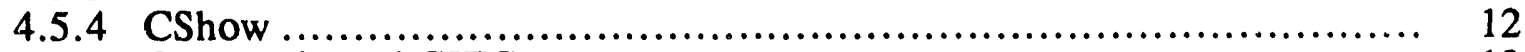

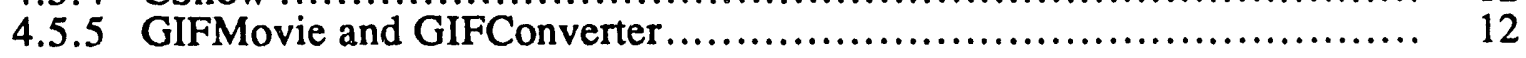

5 Observations.............................................................. 14

5.1 Enhanced Visualization ...................................................... 14

5.2 Portability and Convenience..................................................... 14

5.3 Compatibility of DOS Version of Slide Show ............................. 14

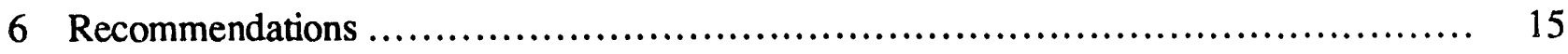

6.1 Applications Research ................................................ 15

6.1.1 Comprehensive Database Documentation................................ 15

6.1.2 GRASS Application Documentation ..................................... 15

6.2 Technical Research ....................................................... 16

6.2.1 Improving Conversion Capabilities and Diversifying Image

Enhancement Techniques............................................. 16

6.2.2 Improving Compression Techniques.................................. 16

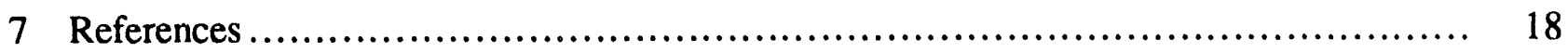

Appendix A File Names and Descriptions for Videographic GIS Database

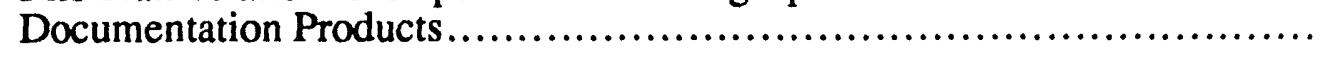




\section{Contents (Cont.)}

Appendix B CShow Display Software Documentation: Instructions for Using CShow by CompuServe ............................................ 23

Appendix C DOS-Compatible Documentation Product Diskette Contents .............. 27

Appendix D GIFMovie Software Documentation...................................... 29

Appendix E Macintosh-Compatible Documentation Product Diskette Contents......... 31

\section{Figures}

1 GRASS-Generated Data Layer and Corresponding Shaded-Relief Map............. 8

$2 \quad \mathrm{~K}$-Factor Composite Image ................................................ 9

3 Geology Layer in Perspective View.......................................... 9 


\title{
Videographic GIS Database Documentation Project for Ft. Knox, Kentucky: Planning and Process
}

\author{
by
}

\author{
R.G. Sullivan and M.C. Vogt
}

\begin{abstract}
The Geographic Resource Analysis Support System (GRASS), a geographic information system (GIS), has been fielded at approximately 50 U.S. Army training installations as a land-management decision-making tool. Use of the GRASS GIS requires the compilation of numerous digital maps of environmental parameters, including elevation, soils, and hydrography. One of the uses of the GRASS GIS is to create digital graphic images that portray geographic data. A recently emerging technology called computer videographics can be used to graphically enhance GRASS-generated images, thereby creating new ways to visualize GRASS analysis results. Furthermore, videographic tools can be used to convert GRASS images into standard graphic file formats that can be displayed on low-cost personal computers, using inexpensive application software to view the images. The pilot project described in this report demonstrated the capability of videographic tools to enhance GRASS imagery through improved graphic visualization (including three-dimensional visualization) and enhanced labeling. The project also developed self-running demonstrations of GRASS imagery from Ft. Knox, Kentucky, on DOS- and Macintosh-compatible diskettes, using lowcost slide-show software to display the images.
\end{abstract}

\section{Introduction}

The Geographic Resource Analysis Support System (GRASS) was developed in the early 1980s by the U.S. Army Construction Engineering Research Laboratory specifically to support the management of land resources at U.S. Army installations. GRASS has been implemented as part of several programs to support installation facility managers, including the Integrated Training Area Management (ITAM) program, a comprehensive land-management program consisting of six initiatives addressing various aspects of training-land resource management. One of the ITAM initiatives calls for implementation of computerized decision-support systems, such as GISs. GRASS has been implemented, or is planned for implementation, at over 75 Army installations in the United States and at several more installations in Europe.

Implementing GRASS at an Army installation requires compilation of numerous data layers that will be used as input to subsequent GRASS analyses. Basic data, such as elevation, soil type, rainfall, and land cover, may be used in many different types of analyses and are often compiled 
before, or soon after, GRASS implementation at a particular installation. These data are usually compiled, at least in part, by the U.S. Army Construction Engineering Research Laboratories (USACERL) and are given to each installation at the time of implementation. In some cases, there may be as many as 50 base data layers compiled for an installation. In order for personnel to become familiar with the compiled data layers for their installation, a bound volume containing a map of each data layer available for the installation is made by USACERL and given to installation personnel. This volume serves as a reference for installation personnel, but it also is intended to help installation personnel become familiar with the capabilities of GRASS itself, as applied to their specific installation. However, these volumes of data layers require considerable labor to develop and constant updates.

Because it is desirable to familiarize installation personnel with GRASS capabilities as quickly and as fully as possible, additional means are being explored for documenting GRASS databases for training installations. Computer videographic technology has been identified as one potential means to present GRASS data to installation personnel. Videographics can be used to enhance visualization of GRASS images, to ensure high graphic quality for imagery, and to present images in a format that engages viewers' interest without overwhelming them with complexity.

The Videographic GIS Database Documentation project was initiated in 1991 for the purpose of demonstrating the use of videographic technology to produce documentation of a GRASS GIS database for a U.S. Army training installation. Ft. Knox, Kentucky, was selected as the installation for this demonstration project, both because it recently began implementation of GRASS as part of the ITAM program and because numerous GRASS data layers have already been compiled for Ft. Knox. 


\section{Background}

USACERL, locaied in Champaign, Illinois, is responsible for development of the ITAM program, which is being implemented throughout the Army. The ITAM program seeks to develop standard land management strategies for Army installations and to exploit advanced technologies to implement those strategies (Goran and Finney 1991). The six major thrusts of ITAM are (1) documentation and monitoring of land resources over time, (2) integration of training requirements with land-management strategies, (3) provision of environmental awareness education to troops, (4) implementation of structural renovation and runoff control technologies, (5) rehabilitation and maintenance of vegetative cover, and (6) implementation of computer-based management and scheduling technologies, including the implementation of GRASS for landresource management.

USACERL requested the assistance of the Reclamation Engineering and Geosciences Section of the Energy Systems Division at Argonne National Laboratory (ANL) in the development of a videographic GIS database documentation product for Ft. Knox. Argonne was selected because of its expertise in applying videographic technology to Army training-land management problems as part of the training requirements integration thrust of the ITAM program.

\subsection{Computer Videographic Technology}

Computer videographic technology is a general term to describe systems of computer hardware and associated software that can be used to create and/or manipulate digital graphic images. Videographic technology can include mainframe computers, workstations, or personal computers (PCs); various types of software; and hardware associated with the production of digital images, such as image scanners, digital printers, and videotape recorder controllers.

Videographic technology is in its infancy, only appearing at the personal computing level in the early 1980s, an event which helped spark an explosion of development of videographic hardware and software. Videographic systems are widely employed to produce high-quality graphics for such purposes as movies, television and magazine advertising, desktop publishing, and scientific visualization.

Videographic software includes "paint" packages that are used to create images, much as a traditional artist paints canvases, and "photo-retouching" packages that can be used to alter existing images which have been scanned into digital format. "Animation" packages can be used to create animated sequences of images that can eventually be transferred to videotape. 


\subsection{Videographic Applications in Army Training-Land Management}

Videographic technology has been applied to Army land-resource management problems as part of the ITAM program since 1989, when it was used to produce simulations of visual impacts at training areas through a technique similar to photo-retouching (Sullivan et al. 1989). Videographic technology was first used to enhance GRASS imagery in 1990 (Sullivan 1991).

Videographic systems used for the ITAM program at USACERL and ANL have primarily utilized IBM-compatible personal computers running under the DOS operating system. The component that distinguishes videographic systems from ordinary PCs is the videngraphics adapter, a circuit board that processes high-resolution, full-color digital images. The systems at USACERL have employed videographics adapters from Truevision, Inc., a major manufacturer of high-quality graphics cards for both IBM-compatible and Macintosh computers. Images are displayed on red-green-blue (RGB) analog monitors, and images are output by a variety of devices, including thermal-rransfer printers and video printers.

Within the ITAM program, videographic systems have been employed primarily to retouch digitized photographs of damaged training-area land. Videographic paint and retouching software is employed to edit the image of the existing landscape to realistically depict the landscape's appearance after rehabilitation actions (such as revegetation).

Recently, videographic technology has been used to graphically enhance images produced by the GRASS GIS software. The GRASS images can be converted into a format compatible with the videographic software and then altered in various ways through the use of both paint and retouching software. The videographic software tools can be used to enhance visualization of GRASS data, to create more legible labels and text for the GRASS images, and to create highquality graphic output from the images in a wide variety of formats.

USACERL requested Argonne to explore the use of videographic technology to produce graphic documentation of the GRASS database for Ft. Knox, resulting in the Videographic GIS Database Documentation project. This report describes the procedures followed in producing the documentation product. 


\section{Goal, Objectives, and Approach}

The goal of the Videographic GIS Database Documentation project was to demonstrate the use of videographic technology to produce an effective GRASS database documentation product for Ft. Knox. To attain this goal, the following specific objectives were defined:

- Use videographic software tools to document the GRASS GIS data layers in the Ft. Knox spatial digital landscape database;

- Use videographic tools to enhance the general graphic quality of the GRASS images through improved text and labeling;

- Produce the documentation product in a digital format compatible with typical IBM-compatible and Macintosh computers;

- Produce a product of sufficiently small size to fit on fewer than four highdensity floppy diskettes in both DOS- and Macintosh-compatible formats; and

- Produce a product that could be easily installed and run on a PC or Macintosh computer by a person with minimal computer experience, using shareware or public domain display software.

The project was divided into five phases: (1) a project work plan was developed, and the content and format of the documentation product were decided upon; (2) base images of various GRASS data layers were constructed using GRASS; (3) the GRASS images were converted into a digital format compatible with the videographic system; (4) the images were enhanced in various ways using videographic software; and (5) the images were converted into a different digital format and assembled into self-installing, self-executing "electronic slide shows" (i.e., programs that would display images according to a predetermined sequence for a predetermined length of time). 


\section{Description of Effort}

\subsection{Development of the Proifui Work Plan}

The initial step in preparing the documentation product was to decide on subject material and presentation ruethod. The project sponsor suggested focusing primarily on erosion-related data layers, as erosion is a major problem at Ft. Knox and would likely be the subject of future GRASS analyses at the installation. The GRASS data layers selected for enhancement included elevation, land use, range areas, $\mathrm{K}$ factor, $\mathrm{T}$ factor, geology, roads, hydrography, and archaeology.

To show the application of GRASS and videographic visualization tools at different scales, part of the documentation slide show presented visualizations of the entire Ft. Knox installation, while another segment was devoted to visualizations of a small portion of $\mathrm{Ft}$. Knox, specifically the area around Cedar Point in the northeast quadrant of the installation. A third segment showed three-dimensional perspective views of the Cedar Point area, all from a particular viewpoint.

\subsection{Creation of GRASS Base Images}

Having determined the content of the documentation product, the GRASS images that were to be enhanced were generated in GRASS. For most of the data layers, a base image was generated for each of the three segments of the final slide show (i.e., a base image was generated for the whole installation, for the Cedar Point area, and for the perspective view of Cedar Point). No perspective views were generated for the data layers containing linear features (roads and hydrography), and not all of the generated base images were used in the final product. In addition, the GRASS command shade.rel.sh that generates a shaded-relief map of topography was run for each GRASS base image. Generating the shaded-relief map for each type of visualization was critical, as the shaded-relief map is used to create most of the enhanced visualizations.

\subsection{Conversion of GRASS Images to a Videographic-Compatible Format}

The next phase of the project required converting the GRASS base images and the shadedrelief layers into a format compatible with the videographic software used for the project. From a list of several possible file types, the TGA format (from Truevision) was selected as the primary format, because it could be used by all of the software packages under consideration for the project. For comparison purposes, some files were converted to PICT format for proc ssing on the Macintosh platform, using a file-exchange package called PICTure This ${ }^{\mathrm{TM}}$, from FGM, Inc. The PICT format is a standardized Macintosh graphics file format usable by many Macintosh software packages. 


\subsubsection{R.out.tga Flle Conversion}

Base images either of the entire installation or of the Cedar Point area that were not perspective views (i.e., plan views) were converted to TGA format using the GRASS command r.out.tga. This command, which can be run interactively or noninteractively, converts GRASS raster images, or rasterized vectors, into TGA format. The r.out.tga command preserves the spatial resolution of the original data layer. After conversion, the TGA files were transferred to the videographics systems via FTP/Ethernet.

\subsubsection{PBM+ Flle Conversion}

The r.out.tga command cannot convert the GRASS perspective views to TGA format, as they are not GRASS raster images. The perspective views must be output as screen dumps, which are Sun raster files, and then converted to TGA format using $\mathrm{PBM}+$, a file-conversion utility shipped with GRASS. The PBM+ utility first converts Sun raster files into an intermediate format, and then converts the intermediate format file into a TGA format file. The use of screen dumps means that the original spatial resolution of the GRASS data layer is lost, because all screen dumps are necessarily $1,152 \times 900$ pixels in resolution. However, the loss of resolution does not seriously interfere with image enhancement or subsequent steps. PICTure This was used to convert some of the Sun raster files to PICT format for processing on the Macintosh.

\subsection{Image Enhancements}

Videographic enhancements to the GRASS images used in the documentation slide show fall into two classes: (1) overlay of the GRASS data layers on the shaded-relief surface to create shaded-relief maps of the GRASS data and (2) addition of high-quality text, labels, backgrounds, and sketch graphics to the images.

\subsubsection{Shaded-Rellef Enhancements}

Videographic paint packages (Lumena ${ }^{\mathrm{TM}}$, from Time Arts, Inc., or Aldus Photostyler ${ }^{\mathrm{TM}}$ for IBM compatibles; Adobe Photoshop ${ }^{\mathrm{TM}}$ for the Macintosh) allow GRASS data layers to be superimposed onto the corresponding shaded-relief map (see Figure 1). In this overlay process, the colors of the GRASS data layer can be made semitransparent. Because the shaded-relief map generated in GRASS is always a gray-scale image, the overlay process in effect tints the shadedrelief map with the colors of the GRASS data layer. The resulting composite image is a shadedrelief map of the original GRASS data. Figure 2 shows a composite shaded-relief map of $\mathrm{K}$-factor that was created using this overlay process. The composite shaded-relief map allows the 

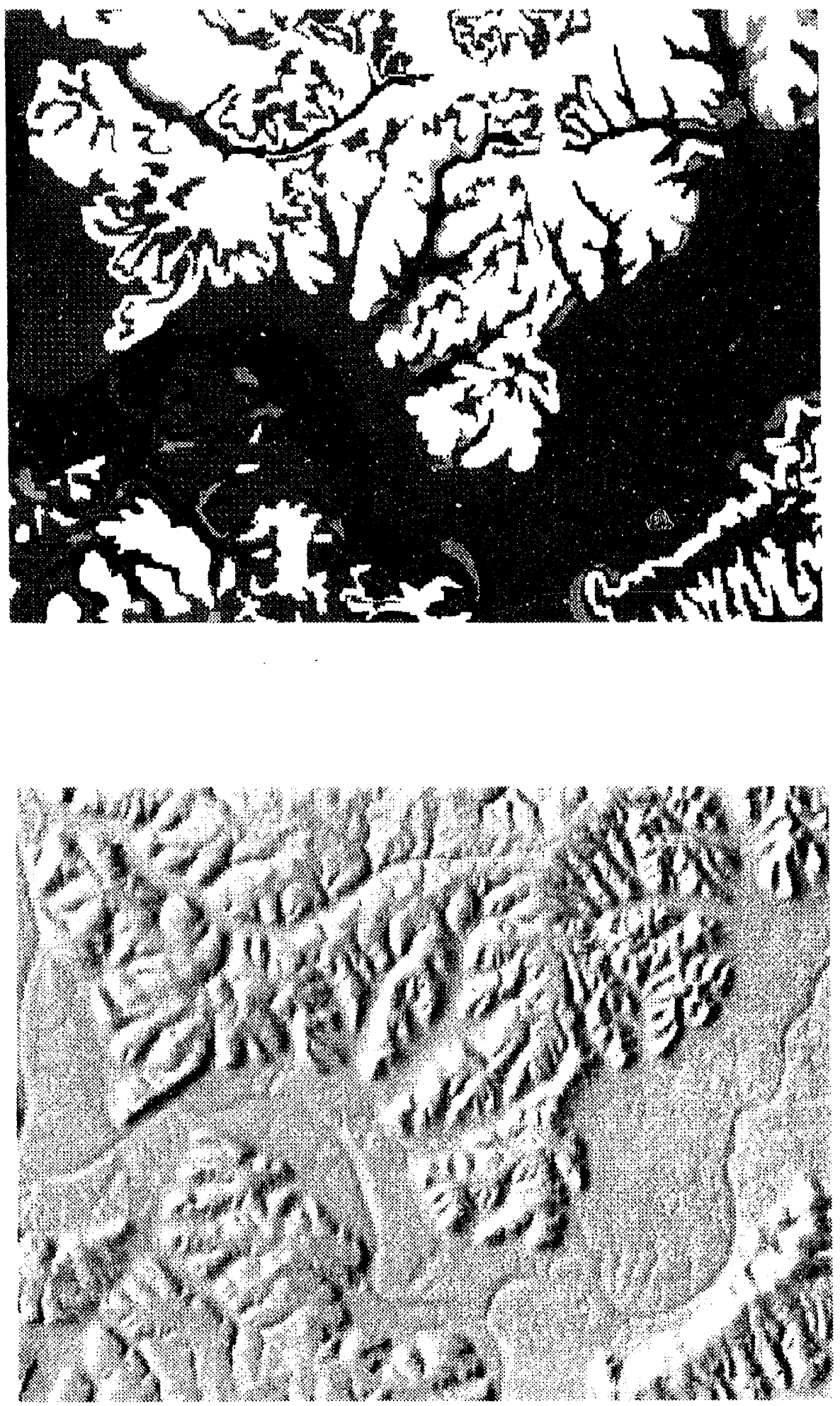

FIGURE 1 GRASS-Generated Data Layer (top) and Corresponding Shaded-Relief Map (bottom) 


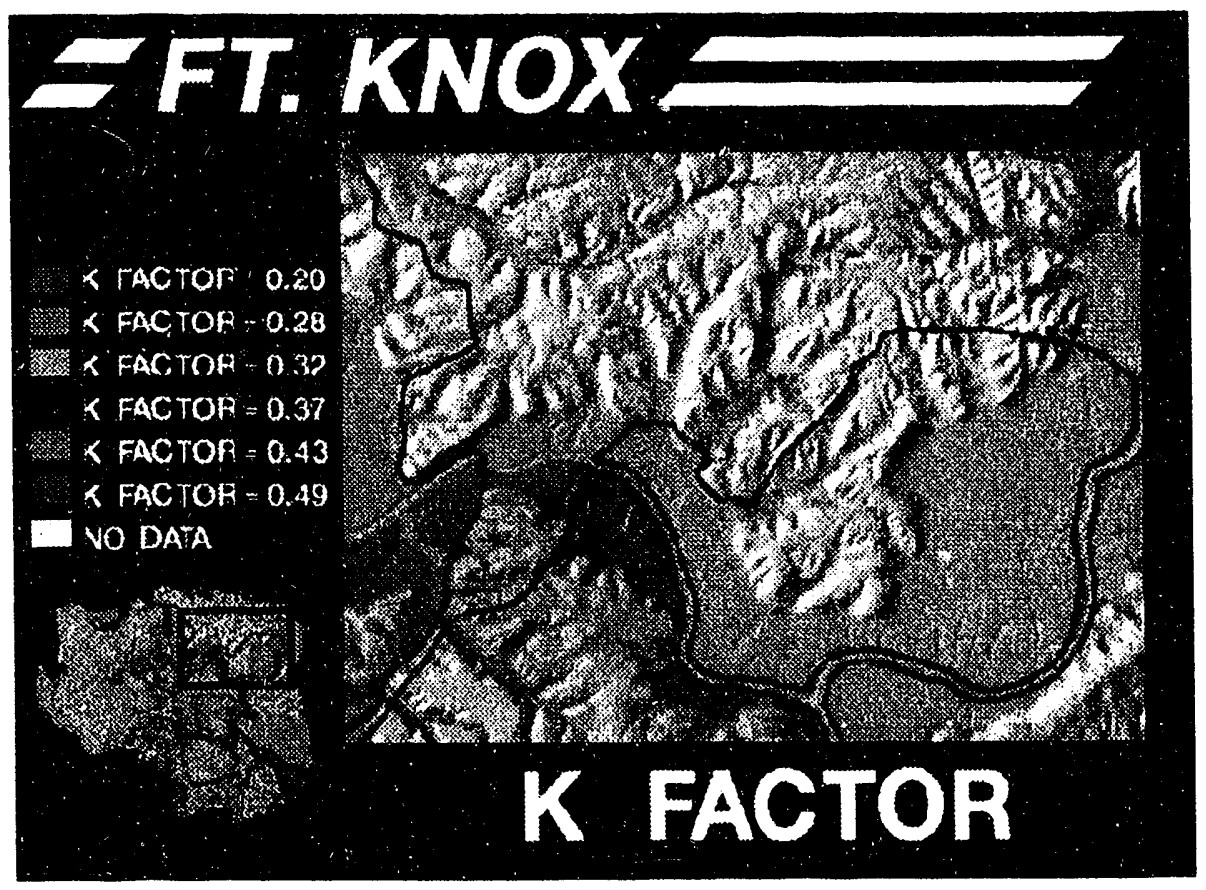

FIGURE 2 K-Factor Composite Image

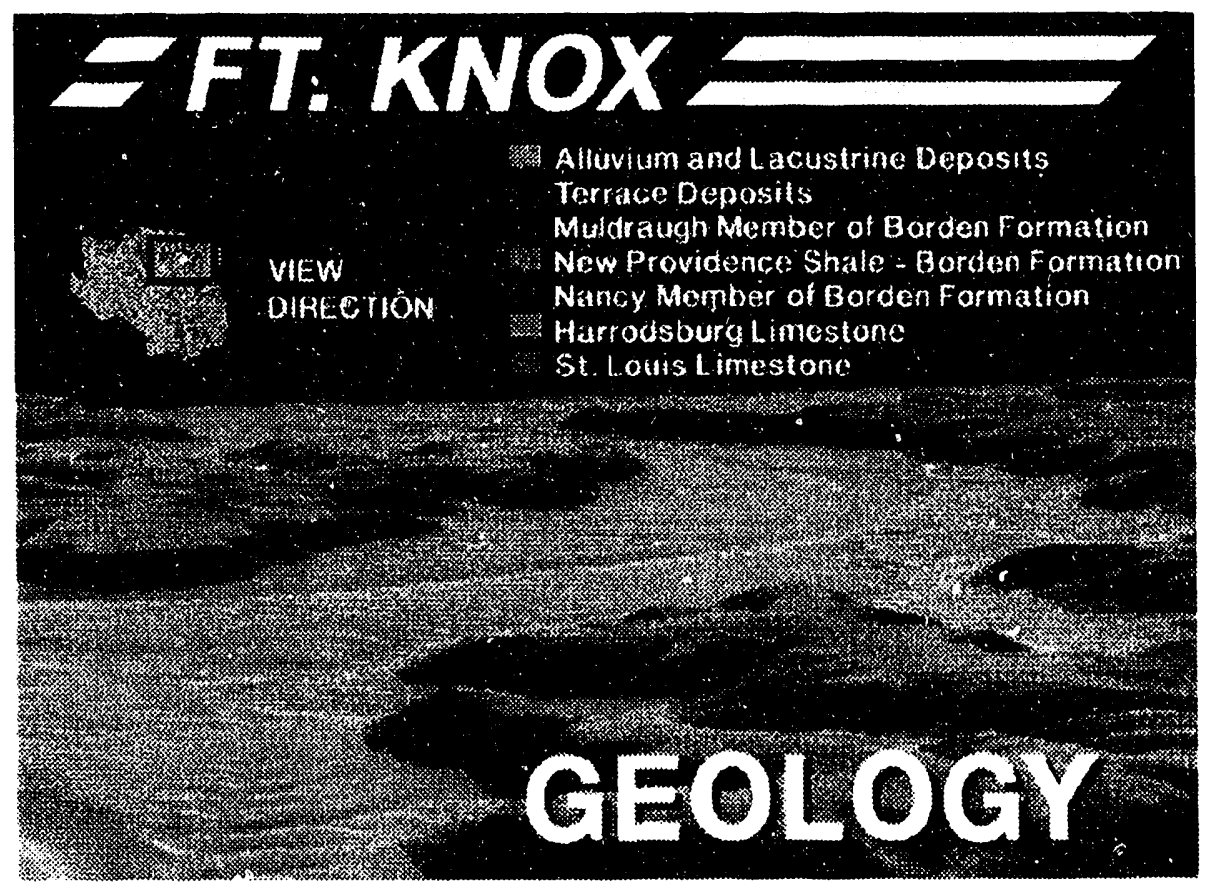

FIGURE 3 Geology Layer in Perspective View 
viewer to associate $\mathrm{K}$-factor values from the original GRASS data layer with the topographic features contained in the original shaded-relief map. Figure 3 shows a perspective view of a shaded-relief map of geology.

Shaded-relief overlays were performed for all of the data layers except the linear features layer (roads and hydrography) in both plan and perspective views. Prior to making the overlays, colors in some of the riginal GRASS layers were changed to increase contrast. This was necessary because overlaying the colored layer onto the gray-scale layer tended to diminish contrast, making it difficult to distinguish between similar colors in the composite overlay.

\subsubsection{Text and Labeling}

After creating the composite shaded-relief maps, a variety of text, labels, and sketch graphics were added to the images. Orientation maps of Ft. Knox were added to many of the images, while a color-gradient backgrcund was added to the plan-view images (see Figure 2). These enhancements were performed using RIO ${ }^{\mathrm{TM}}$ from AT\&T Graphics Software Laboratory. RIO is a resolution-independent, object-oriented software package that allows high-resolution text and sketch graphics to be combined with raster images. RIO allows resolution-independent scenes of composed images to be saved in an internal format and then written out to a TGA file of the desired resolution. The package was extremely useful for composing images, partly because the constructed scenes serve as templates to produce a series of similar images. Because most of the images in the slide show followed one of three formats, the template feature saved considerable time in image preparation and also ensured a more consistent level of graphic quality in the images.

Lumena was used to create the title slide for the perspective view segment. A scanned raster image of a sunset was combined with a soils map of the Cedar Point area to create a montage of the sunset over the composite shaded-relief map. Text was added in RIO.

Lumena was also used to trace the outline of the state of Kentucky for the main title of the slide show. The outline was traced from an atlas with a cordless stylus. RIO was used to resize the outline and place it in the final image.

After preparing several drafts of the images, the final versions were resampled to create files of spatial resolution $640 \times 480$ pixels and reduced in color depth from 24 bits to 8 bits, in order to save space (see discussion below). Files of this size would fit on standard monitcr screens used by most DOS and Macintosh systems. The files were then ready to be converted into the GIF file format used for the slide show. 


\subsection{Creation of DOS- and Macintosh-Compatible Slide Shows}

An objective of this project was to create slide shows that could be displayed on standard computer equipment, would occupy only a few diskettes' worth of memory, and could be run by persons unfamiliar with the display software and perhaps with computers in general. Furthermore, the display software had to be shareware or in the public domain to allow for easy distribution.

\subsubsection{Reduction of Image Color Depth}

Because the TGA and PICT files used were 24-bit color images, they were very large (up to 2.4 megabytes) and required specialized hardware to display. Thus, they were not suitable for the final display format. It was decided to reduce the files to 8-bit color, so that many of them could be fit onto a floppy diskette. This task was accomplished with Photoshop or Photostyler, both of which have excellent dithering algorithms that can reduce color depth from 24 bits to 8 bits with minimal degradation of image quality.

\subsubsection{GIF File Format}

Further reduction in size could be accomplished by selecting a format with built-in image compression. This led to the choice of the GIF format from CompuServe, Inc., a large commercial bulletin-board service. The GIF image format was created by CompuServe, Inc., for distribution of high-quality color graphic images by modem. The GIF format was an excellent choice for final display format for several reasons:

1. The GIF format is strictly standardized and controlled by one entity (CompuServe); thus, there is only one version of the format. The GIF format is consistent across platforms and requires no additional conversion.

2. The GIF format is widely known and used, so that conversion to the format can be accomplished by many paint or retouching packages, as well as dedicated file-conversion packages. Furthermore, numerous shareware and publicdomain software packages are available to display GIF images.

3. The GIF format has an extremely efficient compression algorithm built in, so that GIF files are automatically compressed upon creation. Reducing image files from 24-bit to 8-bit color and saving them as GIF files resulted in an average file size reduction of over $95 \%$, from 2.4 megabytes to 100 kilobytes. 


\subsubsection{Translation of Files to GIF Format}

Translating files from TGA or PICT format to the GIF format was accomplished with Photoshop or Photostyler, as discussed in Section 4.5.1. The resulting 22 GIF files were then ready for inclusion into a slide show composed in the display packages chosen for the DOS and Macintosh platforms. Files for inclusion in the Macintosh version slide show were renamed so that they would play in a particular order, as the display software chosen does not allow scripting the slide show. A complete list of the file names for both the DOS and Macintosh versions, with a brief descriptions of each file, is included in Appendix A.

\subsubsection{CShow}

The DOS-compatible slide show was created with the CShow package from CompuServe. CShow is a shareware program that displays GIF images, allows the user to write a script to display images in a particular sequence, and displays each image for a predetermined time.

A unique feature of CShow is its capability to test the video card present in the computer it is run on, in order to optimize the displayed image quality. This feature is very useful for IBM-compatible computers that may contain a wide variet"' of nonstandardized display cards.

CShow can be run interactively, so that images can be displayed one at a time or a script can be written and triggered by a simple command at the DOS prompt. To simplify the installation and running of the Ft. Knox database demonstration, a small program was written to automate the installation of CShow and the image files. A soript was written to display the Ft. Knox images in a slide show that could be launched by typing a single command at the DOS prompt. If any problems were encountered, CShow contained on-line help, and more extensive documentation could be printed out from files provided with the program. In addition, specific instructions for installing and running the Ft. Knox database slide show were written for distribution with the diskettes. A copy of this documentation is contained in Appendix B.

The final version of the DOS-compatible slide show is contained on three high-density DOS diskettes. All three diskettes contain some of the GIF images, while one diskette also contains the CShow program, the script file, and various support files. A complete listing of the files on each diskette is provided in Appendix C.

\subsubsection{GIFMovie and GIFConverter}

GIFMovie and GIFConverter are two Macintosh-compatible programs for displaying GIF images. GIFMovie is a simple slide-show program that displays selected files in alphabetical order, for approximately 10 seconds each. The user launches the application with a mouse click and then selects the GIF images and the GIFMovie icon with the mouse. After a short delay, a list 
of the files will appear on-screen for several seconds; then the images will display in order for about 10 seconds each.

GIFMovie lacks the user controls of CShow, but it is easier to install and operate. It is a public-domain package that works for most Macintosh computers with color displays. Because Macintosh video display boards are much more highly standardized than DOS-based video boards, there is little need for the video testing function of CShow. Documentation for GIFMovie was enclosed with the diskettes, as well as the TeachText word processor, so that any Macintosh user can read the instructions. A copy of this documentation is contained in Appendix D.

GIFConverter is a program for displaying GIF images on a Macintosh computer one at a time. GIFConverter can also accept other file formats and convert them into GIF images for display purposes. The program is very easy to install and run. All instructions for displaying files are contained in the program menus.

The final version of the Macintosh-compatible slide show is contained on four high-density Macintosh-formatted diskettes. All four diskettes contain some of the GIF images, while one diskette also contains the GIFMovie and GIFConverter applications and various support files. A complete listing of the files on each diskette is provided in Appendix E. 


\section{Observations}

The enhanced images show significant improvement over those typically produced using GRASS alone to generate images. The self-running demonstrations are easy to install and operate.

\subsection{Enhanced Visualization}

The enhancement of the original GRASS images throughi the use of the shaded-relief overlays, as well as the addition of high-quality labels and orientation maps, resulted in images that are effective in communicating geographic data. The shaded-relief overlay is particularly useful to viewers who may be unfamiliar with the particular landscape portrayed in the image, because the shaded-relief overlay provides a depiction of topography as well as the thematic geographic data. Fó- those GRASS users who are familiar with the portrayed landscape, subtle relationships between the geographic data and topography may be noticed for the first time. The inclusion of high-quality labels increases readability and allows the viewer to concentrate on interpreting the image, rather than on trying to interpret the labels.

\subsection{Portability and Convenience}

Had GRASS raster files been used for the database demonstration slide show, the total size for the show would have exceeded 50 megabytes and would have required specialized equipment to display. The slide show as produced requires less than four megabytes and can be displayed on computer equipment that most potential users will have in their offices.

\subsection{Compatibility of DOS Version of Slide Show}

The only significant problem encountered with the demonstration slide show was that the DOS version cannot display high-quality images on every IBM-compatible machine. Useful images require a VGA display capable of $640 \times 480 \times 256$ colors. The CShow program can provide optimal displays on approximately 30 of the most popular video cards; however, because of the plethora of different cards on the market and the constant release of new models, CShow will always be incompatible with some machines. For these machines, CShow has a default display mode that will allow users to view the images, but with a significant decrease in image quality. This problem will require issuers of these types of products to acquire the most recent versions of the display software, which will presumably have more current drivers for popular video cards. The incompatibility problem is much less significant for the Macintosh version because there are fewer video cards for the Macintosh, but it may become significant in the future. 


\section{Recommendations}

The Videographic GIS Database Documentation project for Ft. Knox has demonstrated both the value and feasibility of videographic enhancement of GIS imagery, as well as the use of videographic technology to produce GIS imagery in a format that is convenient and easily distributed. Recommendations for further efforts include additional applications for database documentation, applications for GIS analysis documentation, and technically oriented research to refine the production process.

\subsection{Applications Research}

Recommended applications research includes preparing a more comprehensive database documentation product, as well as exploring the use of electronic slide shows for documenting GRASS applications.

\subsubsection{Comprehensive Database Documentation}

On the basis of the success of this initial effort, a more comprehensive database documentation product using enhanced GRASS imagery should be prepared. This product should be "field tested" (i.e., supplied to potential users). A user evaluation should be performed to assess effectiveness and to solicit ideas for improvements. Cost analyses for producing the product should be performed, in order to assess the feasibility of producing database documentation products for multiple installations.

Documentation products for an installation might cover the entire GRASS database for that installation or might cover only a portion of the database - for example, all layers relating to archaeology. Modules could be developed for each portion of the database, so that size could be kept low. Such products could provide a useful reference to installation personnel who do not have the specialized equipment or knowledge to run GRASS itself.

\subsubsection{GRASS Application Documentation}

The type of product developed for this project has potential for demonstrating applications of GRASS, not only for Army personnel, but for educational and public information purposes. At the installation level, GRASS application demos could be used to brief decision makers at remote locations about land-management issues by using materials far more effective than paper prints. The availability of notebook computers with 8-bit color will facilitate bringing demonstrations to any location. 
GRASS demonstrations on diskette could also be used for educational purposes: to illustrate GIS concepts to persons with no access to GIS equipment or to persons desiring information about a particular application. Because of the small size of the images and their common format, they could easily be incorporated into interactive learning sessions or tutorials.

The demonstrations could be used for public-information purposes, either for presentation to small groups or, with suitable projection equipment, to larger groups. Interactive demonstrations of GRASS applications mixed with photographs, support graphics, and text slides could be used at public meetings or other settings where the public might be interested in learning about decisions based on GRASS analyses.

\subsection{Technical Research}

Technical research issues include improving file conversion capabilities within the GRASS program, diversifying image enhancement techniques, and using better compression techniques to further reduce file size.

\subsubsection{Improving Conversion Capabllities and DiversIfying Image Enhancement Techniques}

One aspect of the current process that needs improvement is the method for converting GRASS files to different formats within GRASS. The current r.out.tga command in GRASS is limited to specifying a file for conversion and a destination file. An improved file-conversion command could allow users to specify output resolution and image compression options and should be able to convert GRASS rasters and screen dumps to multiple output formats, specifically TGA, TIFF (Tag Image File Format), GIF, and PICT. Writing an improved conversion command would save time that would otherwise be spent resampling images to change resolutions and converting files to different formats.

\subsubsection{Improving Compression Techniques}

Image compression played a very important role in producing a product that met requirements for portability. Without image comoression, one diskette would have been required for each image in the show, and the large number of diskettes needed would have been too unwieldy to install.

Attempting to compress images even further presents significant opportunities for improving this documentation product and opening new applications. If greater compression ratios were achieved, more images could be fit onto a given number of diskettes, or fewer diskettes could 
be used. More importantly, the shows might be made small enough to be sent to remote sites via modem. This would greatly improve dissemination of GRASS data for a variety of purposes.

The compression method inherent in the GIF format is a very efficient compression technique. However, this type of compression is a "lossless" technique (i.e., no color data is removed during compression, and the compressed image is identical to the precompression image). Lossless compression techniques are limited in the degree of file compression achievable because of the need to preserve all of the color data from the original image.

Recently, high-quality "lossy" compression techniques have become available. Lossy compression techniques discard some of the original image data, and the compressed image differs slightly from the precompressed image. Lossy compression techniques usually have several degrees of compression that can be specified by the user. For example, the user can select a "best quality" mode that degrades image quality imperceptibly, but still achieves considerably better compression ratios than lossless techniques. If the user selects a lower-quality compression mode, a still higher compression ratio is achieved, but image-quality degradation is also greater.

A lossy compression technique commonly employed is the JPEG method (from the Joint Photographic Experts Group). JPEG features several degrees of compression that still maintain high image quality with high compression ratios. JPEG-compressed images are compatible with many software packages and seem to be the emerging standard. Research in this area should focus on testing such lossy compression techniques as the JPEG to determine the best levels of compression as well as potential file-size savings. 


\section{References}

Goran, W.D., and D. Finney, 1991, "GRASS GIS Critical to Army's Land Management Program," GIS World, 4(9):48-53.

Sullivan, R.G., T.G. Sydelko, and R. Lacey, 1989, "Training Land Design Using Video Imaging Technology," presented at the American Society of Agronomy 1989 Annual Meeting, Las Vegas, Nev., Oct.

Sullivan, R.G., 1991, "Geographic Data Visualization and Communication in Army Training Land Management," presented at the American Society of Agronomy 1991 Annual Meeting, Denver, Colo., Oct. 


\section{Appendix A:}

\section{File Names and Descriptions for Videographic GIS Database Documentation Products}

File Name

kargcerl.gif

katitleg.gif

kargng.gif

kargshg.gif

kaelerdg.gif

kaelehyg.gif

katfg.gif

kakfshg.gif

kititle.gif

kinewrds.gif

kinewhyg.gif

\section{Description}

Introduction slide, USACERL and ANL logos

Scanned and retouched tanks, outline of state of Kentucky showing location of Ft. Knox, image of entire installation with overlaid shaded-relief and elevation layers

Image of entire installation with overlaid land-use designations on shadedrelief layer

Image of entire installation. with overlaid range boundaries on shaded-relief layer

Image of entire installation with overlaid shaded relief on elevation layers and additional overlay of road structure

Image of entire installation with overlaid shaded relief on elevation layers and additional overlay of hydrography

Image of entire installation with overlaid shaded relief on $\mathrm{T}$-factor layers

Image of entire installation with overlaid shaded relief on $\mathrm{K}$-factor layers and additional overlay of range boundaries

Rectangle showing Cedar Point location on image of entire installation with overlaid shaded relief on elevation layers including scanned tanks

Two images: small image of entire installation showing location of Cedar Point area within red rectangle and larger image of Cedar Point area with overlaid shaded relief on elevation layers topped by road structure

Two images: small image of entire installation showing location of Cedar Point area within red rectangle and larger image of Cedar Point area with overlaid shaded relief on elevation layers topped by hydrography 
kiarcsh.g.gif Two images: small image of entire installation showing location of Cedar Point area within red rectangle and larger image of Cedar Point area with overlaid archaeology on shaded relief layers

kingeohy.gif Two images: small image of entire installation showing location of Cedar Point area within red rectangle and larger image of Cedar Point area with overlaid shaded relief on geography layers topped by hydrography

kislopeg.gif Two images: small image of entire installation showing location of Cedar Point area within red rectangle and larger image of Cedar Point area with overlaid shaded relief on slope topped by range boundaries

kintfrng.gif Two images: small image of entire installation showing location of Cedar Point area within red rectangle and larger image of Cedar Point area with overlaid shaded relief on $\mathrm{T}$-factor topped by range boundaries

kinkfrng.gif Two images: small image of entire installation showing location of Cedar Point area within red rectangle and larger image of Cedar Point area with overlaid shaded relief on $\mathrm{K}$-factor topped by range boundaries

k3dtitle.gif Image of Cedar Point area in three-dimensional (3-D) perspective with overlaid shaded relief on soil classes and scanned sunset backdrop

kiarc3dg.gif Three images: small image of entire installation showing location of Cedar Point area in red rectangle on shaded-relief layer; larger image of Cedar Point area showing point of view for perspective image over shaded relief; and largest image of Cedar Point, in 3-D perspective, of shaded relief topped by archaeology sites

ki3dgeog.gif Three images: small image of entire installation showing location of Cedar Point area in red rectangle on shaded-relief and geology layers; larger image of Cedar Point area showing point of view for perspective image over shaded relief on geology layers; and largest image of Cedar Point, in 3-D perspective, of shaded relief on geology layers

ki3dtfg.gif Three images: small image of entire installation showing location of Cedar Point area in red rectangle on shaded-relief and T-factor layers; larger image of Cedar Point area showing point of view for perspective image over shaded relief on T-factor layers; and largest image of Cedar Point, in 3-D perspective, of shaded relief on $\mathrm{T}$-factor layers 
ki3dkfg.gif Three images: small image of entire installation showing location of Cedar Point area in red rectangle on shaded-relief and K-factor layers; larger image of Cedar Point area showing point of view for perspective image over shaded relief on K-factor layers; and largest image of Cedar Point, in 3-D perspective, of shaded relief on $\mathrm{K}$-factor layers

kcopy.gif Further information slide, copyright notice 


\section{Appendix B:}

\section{CShow Display Software Documentation: Instructions for Using CShow by CompuServe}

\section{General Instructions}

1. Transfer CShow system files and image files from diskettes to your hard drive.

2. Install CShow video drivers and support files.

3. Launch CShow application.

\section{Transferring Files}

a. Insert the disk labeled ANLDEMO1 into your floppy drive. Change current drive to that floppy. Type "INSTALL1 [drive]: <cr>" (where [drive] is a valid hard drive C: D: E: etc. capable of storing 3 Megs of images). Allow the new directory "ANLDEMO" to be created and the files copied from the floppy to it.

b. Insert the disk labeled ANLDEMO2 into the floppy drive. Type "INSTALL2 [drive]: <cr>". Allow these files to be copied to the new directory.

c. Insert the disk labeled ANLDEMO3 into the floppy drive. Type "INSTALL3 [drive]: <cr>". Allow these files to be copied to the new directory.

This should complete the file transfer. The following files should be in a new subdirectory called "ANLDEMO":

CSHOW.SCR

SCRIPT.EXE

CSHOWI.EXE

CSHOW.EXE

README.EXE

CSHOW.DOC

$<$ Various other "picture" files> (e.g., K3Dtitle.gif)

$<$ Various other support files> (e.g., ANLDEMO.SCR). 
Put the disks away in a safe place and go on to the next step of setting up CShow.

\section{Installing Video Drivers}

a. As with any video display software, CShow should be tailored for each platform's particular video adapter. This is done in CShow by running CSHOWI.EXE. Type "CSHOWI <cr>". You will jump into a menu-driven window to select colors for CShow's interface. You can just hit a <cr> all through the first screen's settings, and they will remain at defaults (unless you wish to change some color/s).

b. The second screen that appears allows you to choose a video adapter from a list of about 30 . As indicated on screen, you can hit the F1 key to take a peek at your system's video code to give you a hint as to which adapter to use if you are not certain. After choosing an adapter by typing in its number, you will be asked to test that adapter's possible display modes. Follow the simple instructions of hitting $<\mathrm{cr}>$ and answer $\mathrm{Y} / \mathrm{N}$ as to whether an acceptable picture was displayed for each test. If you have chosen the right type (it does not have to pass all the video tests, and it should not do so unless it has over 1 megabyte of on-board video RAM), it will ask you if you wish to install that adapter. You can choose no and test others till you find one with the most possible modes that generate good, clean pictures.

c. The third screen in the setup selects a printer driver. Enter in your default printer's number and hit $<\mathrm{cr}>$ to continue.

d. The fourh screen lets you se'ect buffer settings and file display settings. Hit $<c r>$ for each of the lines to leave all at their defaults. The last selection will ask if you wish to calibrate delays. Enter yes. It will run a very short test of your system's clock speed and ask you to hit one last $<c r>$ io complete CShow installation. Now you are ready to use CShow to view files.

Once video installation is complete it need not be repeated on that computer. If the software is installed on another computer, it will have to be performed again.

\section{Launching CShow Application}

a. You can run CShow as a slide shower to present a prearranged slide show by typing "CSHOW @ filename.scr <cr>" where filename.scr is a text file listing the slide show.

- For the ANLDEMO, type "CSHOW @ANLDEMO.scr<cr>".

- After each picture displays, hit the space bar to proceed. 
- To exit the show, type "ESC".

b. You can also run CShow interactively, opening and displaying any possible images in a variety of modes with options by typing "CSHOW <cr>". When in the interactive mode, it is useful to type the following short command sequence "F5 C <cr>". Tinis changes the way files/directories are displayed and allows easy selection of files for viswing. To view a file, simply use arrow keys to position the cursor to highlight the file, and hit $<\mathrm{cr}\rangle$. The file will be selected and information about it displayed. Display modes are selected by hitting the designated special character shown. Bold or highlighted characters are optimal modes. Favored display modes special to a video adapter are listed in the bottom portion of the window. These modes display the best possible picture on a particular video adapter. Typically "W" represents the sharpest $640 \times 480 \times 256$ color image on most adapters.

c. The ESC is used throughout CShow to escape or cancel operations. ESC can be used to halt the display of an image or to basktrack through ihe window/menu system.

d. The PAGEUP and PAGEDOWN keys can be used to brighten or darken a picture that has been displayed.

e. The F1/F2, F3/F4, F5/F6 keys can be used to alter the red, green, or blue content of a displayed picture.

f. The arrow keys can be used to "pan" around a picture that is too big to display on the visible screen. Such images can fit in CShow's video buffers, but are too big to display without panning.

g. To leave CShow, hit ESC several times to exit each layer of control, finally exiting CShow itself.

h. If problems are encountered or more assistance is required, launch CShow's complete documentation/information utility by typing "README.EXE". This enters a complete interactive documentation application that allows searching and printing of complete CShow documentation. 


\section{Appendix C:}

\section{DOS-Compatible Documentation Product Diskette Contents}

Disk 1

install1.bat

kargcerl.gif

kiarc3dg.gif

kiarcshg.gif

kinewhyg.gif

kinewrds.gif

kingeohy.gif

kinkfrng.gif

kintfrng.gif

ktitle.gif

knoxdocs.txt

readme.anl

\section{Disk 2}

install2.bat

kaelehyg.gif

kaelerdg.gif

kakfshg.gif

kargng.gif

kargshg.gif

katfg.gif

katitleg.gif
Notes

Makes directory on designated drive, installs disk contents

Image file

Image file

Image file

Image file

Image file

Image file

Image file

Image file

Image file

General information about images

Installation instructions

\section{Notes}

Installs disk contents

Image file

Image file

Image file

Image file

Image file

Image file

Image file 
Disk 3

install3.bat
k3dtitle.gif
kcopy.gif
ki3dgeog.gif
ki3dkfg.gif
ki3dtfg.gif
kislopeg.gif
anldemo.scr
cdosdemo.scr
ddosdemo.scr
edosdemo.scr
fdosdemo.scr
gdosdemo.scr
cshow.scr
fonts.drv
printer.drv
video.dat
video.drv
readme.exe
script.exe
cshow.cfg
cshow.exe
cshowi.exe

\section{Notes}

Installs disk contents

Image file

Image file

Image file

Image file

Image file

Image file

CShow script file

CShow script file

CShow script file

CShow script file

CShow script file

CShow script file

CShow script file

CShow fonts driver

CShow printer driver

CShow video board information

CShow video driver

CShow full documentation

CShow script execution program

CShow configuration file

CShow program

CShow video board testing program 


\section{Appendix D:}

\section{GIFMovie Software Documentation}

The following text is contained in the file Readme.ANL in the DOCS folder on disk 1:

\section{Instructions for using GIFMovie}

\section{General Instructions}

1. Transfer documentation and image files from diskettes to your hard drive.

2. Launch GIFMovie application to see slide show.

3. Launch GIFConverter application to view single images.

\section{Transferring files}

a. Insert the disk labeled ANLDEMO1 into your floppy drive and drag-copy the folder titled ANLDEMO and the folder titled DOCS to your hard drive. Do not put DOCS folder into the ANLDEMO folder; it will interfere with how GIFMovie operates.

b. Insert the disk labeled ANLDEMO2 into your floppy drive and drag-copy all the files found there into the ANLDEMO folder initially copied to your hard drive.

c. Insert the disk labeled ANLDEMO3 into your floppy drive and drag-copy all the files found there into the ANLDEMO folder initially copied to your hard drive.

d. Insert the disk labeled ANLDEMO4 into your floppy drive and drag-copy all the files found there into the ANLDEMO folder initially copied to your hard drive. 


\section{Launching GIFMovie}

Begin by opening the ANLDEMO folder that was copied to your hard drive. Select all the items in that folder; this should include the *.gif files and the GIFMovie application and no others. Go to the File menu and choose "Open". The movie should automatically begin by displaying a list of the images it will present. It will then display each picture, in sequence, for about 10-15 seconds each. When the show is done, it will halt on the last picture. A "mouse-click" will cancel the show.

\section{Running GIFConverter}

The GIFConverter application allows the files to be viewed individually. After the application is launched, the display can be set for color, gray scale, or monchrome. After files are opened, they can be rotated, transformed, and printed. All GIFConverter commands are explained in its menus. 


\section{Appendix E:}

\section{Macintosh-Compatible Documentation Product Diskette Contents}

Disk_1

ANLDEMO

02katitleg.gif

03kargng.gif

04kargshg.gif

09kititle.gif

DOCS

GIFConverter 2.2.10

Knoxdocs.txt

Readme.anl

TeachText

\section{Disk 2}

05kaelerdg.gif

06kaelehyg.gif

v07katfg.gif

08kakfshg.gif

10kinewrds.gif

12kiarcshg.gif

\section{Notes}

Folder for images

Image file

Image file

Image file

Image file

Folder for documents, programs

Single GIF image display program

General information about images

Instructions for GIFMovie program

Word processor for reading docs

\section{Notes}

Image file

Image file

Image file

Image file

Image file

Image file 
Disk 3

11kinewhyg.gif

13kingeohy.gif

14kislopeg.gif

15kintfrng.gif

16kinkfrng.gif

GIFmovie1.1v

Disk 4

01kargcerl.gif

17k3dtitle.gif

18kiarc3dg.gif

19ki3dgeog.gif

20ki3dtfg.gif

$21 k i 3 d k f g . g i f$

$22 k c o p y . g i f$
Notes

Image file

Image file

Image file

Image file

Image file

GIF slide show program

Image file

Image file

Image file

Image file

Image file

Image file

Image file 

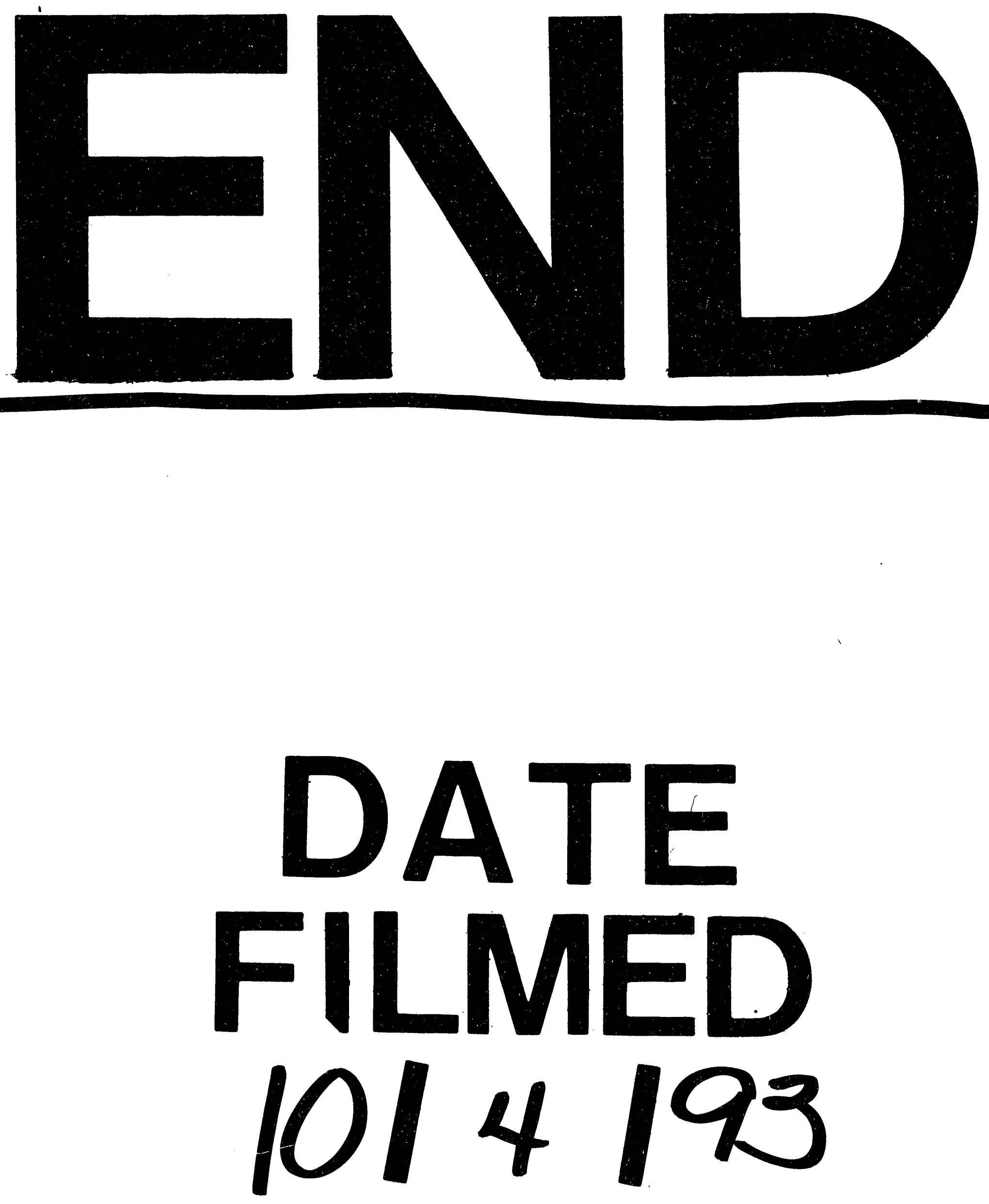
